TRANS · núm. 25.2021

MISCELÁNEA $\cdot 413-431$

INTERPRETACIÓN

Las relaciones entre memoria de trabajo y actividad interpretativa han sido evaluadas con diferentes instrumentos de exploración psicolingüística. Se propone en este estudio una versión adaptada de una prueba de retención de palabras con dos versiones: una en lengua nativa, español, y otra en lengua extranjera, francés, administrada a sujetos que se inician en la práctica de la interpretación simultánea. El estudio aporta y somete a discusión los fundamentos metodológicos de la prueba y algunos resultados que confirman su utilidad a la hora de evaluar capacidades de memoria de trabajo implicadas en la referida práctica.

PALABRAS CLAVE: cognición, memoria de trabajo, capacidad de retención, interpretación simultánea, conocimiento de lengua extranjera.

\title{
Memoria de trabajo e interpretación simultánea: Propuesta de un test adaptado de retención de palabras*
}

\author{
SAIDA AnsSARI-NaIm \\ Carlos HeRnández Sacristán \\ Universidad de Valencia
}

\section{Working memory and Simultaneous Interpreting: a proposal of an adapted test of word retention}

The relationships between working memory and interpreting have been evaluated with different instruments of exploration from psycholinguistics. In this study, an adapted version of a word retention test is proposed with two versions: one in the native language, Spanish, and another in the foreign language, French, administered to beginner students in interpreting. The study provides and discusses the methodological foundations of the test and some results that confirm its usefulness when assessing working memory capacities involved in the referred practice.

KEY WORDS: cognition, working memory, listening span, simultaneous interpreting, foreign language proficiency.

\footnotetext{
* El presente estudio forma parte de las actividades del proyecto de investigación FFI2017-84951-P "Dimensiones metacognitivas en la adquisición, las alteraciones clínicas del lenguaje y la práctica interlingüística", que financia el Ministerio de Ciencia, Innovación y Universidades.
} 


\section{1. INTRODUCCIÓN}

La memoria de trabajo (working memory) se propone inicialmente por Baddeley y Hitch (1974) como factor implicado en el control ejecutivo de la conducta humana y, más específicamente, de la conducta verbal. Baddeley y Hitch (1974) y Baddeley $(1986,1996,2012)$ proponen este constructo psicológico para diferenciar, dentro de las actividades de la memoria a corto plazo (short term memory), aquellas en que a la tarea de simple retención on-line de unidades o estímulos se suma alguna otra tarea de procesamiento. La memoria de trabajo es una capacidad que se integra en el dominio cognitivo más general que Baddeley (1996) denominaba ejecutivo central, donde se incluyen procesos psicológicos superiores como la inhibición de distractores, el control atencional, la coordinación de tareas, la planificación de una tarea guiada por un objetivo externo, entre otros ${ }^{1}$.

El tema de la memoria de trabajo viene interesando por sus relaciones con la práctica interpretativa desde estudios que podemos considerar pioneros como los de Padilla (1995), Padilla et al. (1995), y Padilla y Bajo (1998). Pueden citarse, con posterioridad a estos, otros estudios como los de Padilla Adamuz (2002), Christoffels (2004), Köpke y Nespoulous (2006), Timarová et al. (2008), Hodákova (2009), Tzou et al. (2012), Timarová (2014), Aguirre Fernández-Bravo (2015), Hiltunen et al. (2016), entre otros. A la especificidad de la memoria de trabajo, en el estudio sobre sus relaciones con la práctica interpretativa, se refería Timarová (2008: 1) con las siguientes palabras: «The crucial difference was that short-term memory was a simple store for information, whi-

\footnotetext{
I Véase Baddeley, Eysenck y Anderson (2009) para una versión más actualizada sobre el ámbito específico de la memoria de trabajo dentro del dominio más general de la facultad de la memoria.
}

le the more modern concept of working memory is assumed to be a cognitive component combining storage, processing and executive control of the cognitive processes at hand». Una tarea sería, por ejemplo, la simple retención de una secuencia de números que se nos ofrece como estímulo y donde $7( \pm 2)$ unidades determinan un límite común de la capacidad de almacenaje. Otra tarea, bien diferente, sería retener on-line las palabras de una oración que estamos escuchando, agrupándolas según las reglas sintácticas, para atribuir finalmente un significado a lo que se nos dice. Una memoria a corto plazo en sentido estricto, que supone un mero almacenaje de datos, representa una actividad cognitiva disociada de otras. Este tipo de actividad no es la más común en la conducta humana, que suele ligar, por el contrario, almacenaje online con algún tipo de procesamiento. Esto es, el almacenaje se suele encontrar intencional o funcionalmente guiado por alguna otra actividad en la que nos implicamos.

Esta es la situación común en el almacenaje online de datos verbales para la comprensión oral o la comprensión lectora de textos, pero también para la producción del lenguaje o la escritura. Es inequívocamente también el caso cuando la tarea a la que sirve el almacenamiento consiste en una actividad de mediación interlingüística $y$, muy especialmente, una actividad como la interpretación simultánea, en la que a la comprensión oral en lengua origen se suma la producción oral en lengua meta. Timarova et al. (2014: 143) señalan que, aunque no podemos afirmar de manera genérica que los intérpretes dispongan de mejores capacidades de memoria de trabajo que los no intérpretes, parece clara, sin embargo, la relación entre memoria de trabajo y calidad de la práctica interpretativa. Se destaca, por otra parte, en este estudio la relación entre memoria de trabajo y el control atencional. 
Un antecedente que focaliza también la idea de relacionar memoria de trabajo con otras funciones psicológicas superiores, lo encontramos en el referido estudio de Köpke y Nespoulous (2006). Las actividades que podemos considerar específicas de la memoria de trabajo, se ligan en este estudio a otro tipo de tareas cognitivas asociadas o no al procesamiento lingüístico a la hora de explicar la calidad en la práctica interpretativa. Conviene destacar dos aspectos de los resultados que nos ofrecen estos autores. Por una parte, solo aquellas pruebas específicamente relacionadas con el procesamiento del lenguaje correlacionan de manera significativa con la calidad en la práctica interpretativa. No solo, así pues, para la memoria de trabajo, sino también para otro tipo de tareas que implican a funciones psicológicas superiores, $\mathrm{y}$ en particular para los procesos atencionales, resulta relevante la especificidad del ámbito de aplicación, el lenguaje en este caso. Por otra parte, en el estudio que comentamos se comparan los resultados de dos grupos de sujetos, intérpretes en proceso formativo e intérpretes profesionales, y se concluye que la predictibilidad del factor memoria de trabajo respecto a la calidad en la práctica interpretativa, es mayor para los aprendices que para los intérpretes profesionales.

Aunque este último resultado pueda parecer contraintuitivo, admite una posible explicación. En Anssari-Naim (2021) se someten a contraste tareas propias de memoria de trabajo con componentes actitudinales a la hora de predecir calidad en la práctica interpretativa en estudiantes. Se comparan en concreto los resultados de una prueba de listening span, como representativa de tareas propias de memoria de trabajo, y un test de autoeficacia $^{2}$, como representativo de los componentes actitudinales. En este caso, las correlacio-

\footnotetext{
2 Test inicialmente propuesto por Bandura (1995) con el que se trata de medir la visión subjetiva que tiene un individuo sobre su capacidad de afrontar y resolver problemas prácticos. Existen diferentes versiones adaptadas del mismo.
}

nes positivas y significativas solo se observan entre calidad en la práctica interpretativa, evaluada con una prueba específica, y tareas de memoria de trabajo. Entre calidad en la práctica interpretativa y autoeficacia las correlaciones no son significativa o son, incluso, negativas. Dado que autoeficacia se ha considerado un factor actitudinal que correlaciona positivamente con la práctica profesional de la interpretación en otros estudios (Jiménez Ivars et al., 2014; Lee, 2014), la explicación de los resultados que acabamos de comentar podría estar, según se comenta en Anssari-Naim (2021), en que las estrategias mentales puestas en juego en la práctica interpretativa cambian sustancialmente cuando pasamos del contexto del aprendizaje inicial al de la práctica experta. Mientras que la relevancia de los factores cognitivos, que podemos denominar operativos, es muy superior a los actitudinales en los estudiantes, la situación posiblemente se invierta en la práctica profesional. En esta última el componente actitudinal, que deriva de la experiencia, va ganando más peso relativo en comparación con los factores operativos como la memoria de trabajo.

\section{INSTRUMENTO PARA LA MEDIDA DE LA CAPACIDAD RETENTIVA DE PALABRAS: LIS- TENING SPAN}

La memoria de trabajo puede evaluarse de muy diversas formas. Una primera discusión que cabría realizar es si podemos definirla como un factor genérico o debe considerarse específica de determinadas tareas, tal como acabamos de comentar (Turner y Engel, 1989). En este punto concreto, el debate se encuentra realmente abierto, aunque se admite, en general, una posición intermedia por la que se entiende que para la memoria de trabajo son compatibles un componente genérico y otro $\mathrm{u}$ otros relativos a tareas específicas. En cualquier caso, un estudio global de las capacidades de memoria de trabajo resulta 
416 experimentalmente poco viable, y lo usual es que la memoria de trabajo se evalúe a partir de tareas concretas que se presuponen representativas de la capacidad general. Este es el caso particular de las tareas que se refieren a la capacidad retentiva de palabras escritas, reading span, $\mathrm{u}$ oralmente formuladas, listening span. Nos referiremos en lo que sigue a este tipo de tareas, inicialmente propuestas por Daneman y Carpenter (1980).

El estudio de Daneman y Carpenter (1980) tenía por objetivo evaluar el papel que desempeñan las capacidades de memoria de trabajo a la hora de explicar diferencias individuales en la comprensión lectora. Daneman y Carpenter (1980) proponen para el primer experimento de su estudio un test de reading span con el que se evalúa la capacidad de retención de las últimas palabras de una serie de oraciones que los sujetos leen en voz alta. El test resulta relativamente sencillo de administrar y con un sistema de puntuación fácil también de aplicar. Este sistema tiene que ver con el número de oraciones que el sujeto es capaz de retener, lo que define su reading span. La operación de memoria que se requiere es, por lo que acaba de afirmarse, altamente selectiva (recordar solo la última palabra de series de oraciones que el sujeto lee en voz alta). Uno puede preguntarse por la significatividad de esta tarea para una operación que se presupone mucho más compleja, como es la de la comprensión lectora, en la que las variables que tienen que someterse a memoria y/o procesamiento son por supuesto algo más que la última palabra de las oraciones que se leen.

Siendo conscientes de este hecho, Daneman y Carpenter (1980) correlacionan los resultados del reading span con otras pruebas más específicas de comprensión lectora. Nos referimos en particular a la capacidad de los sujetos para recordar hechos referidos en los textos leídos, la capacidad de identificar referentes de pronom- bres cuyos antecedentes han aparecido en oraciones previamente leídas, así como los resultados que tiene el estudiante en el componente verbal de la prueba general de selectividad para los estudios que se conoce como SAT (Scholastic Aptitude Test) ${ }^{3}$. Los resultados del reading span correlacionan de manera positiva y significativa con los resultados obtenidos en las otras tres medidas, esto es, la capacidad de recordar hechos relatados, la de identificar referencia de pronombres y los resultados que obtiene el estudiante en el componente verbal de la prueba $S A T$. De esta forma quedaría probada la validez del test de reading span en tanto que indicador de las habilidades de comprensión lectora, pese al carácter aparentemente muy selectivo de la operación de memoria que se solicita de los sujetos evaluados.

Adicionalmente, y a los simples efectos del contraste, a los participantes en el experimento se les pidió también que realizaran una tarea de word span en la que lo requerido es simplemente que se recuerden series de palabras, pero desligadas en este caso de todo contexto sintáctico. La operación que se solicita implicaría a las capacidades de memoria a corto plazo, pero no propiamente a la memoria de trabajo, ya que se presupone que no habría ningún tipo de procesamiento asociado a la operación de recuerdo. Los resultados de esta prueba de word span también correlacionan positivamente con las pruebas de recuerdo de hechos, identificación pronominal y componente verbal del $S A T$, pero lo hacen de manera estadísticamente no significativa. En definitiva, una prueba como el reading span (en la que se solicita recordar palabras en contexto sintáctico) puede considerarse indica-

3 Se trata de una conocida prueba utilizada para tomar decisiones sobre admisión de los estudiantes a los estudios universitarios en Estados Unidos, donde se contienen secciones referidas a la capacidad matemática y a la capacidad verbal. 
tiva de capacidad de comprensión lectora, pero no puede decirse lo mismo para una prueba de word span (en la que se solicita recordar palabras fuera de todo contexto sintáctico).

Existe un interés especial en garantizar que la prueba de reading span se encuentra dentro del espacio cognitivo propio de la memoria de trabajo, y no es una simple operación de memoria a corto plazo. Algunos aspectos relativos al tiempo de exposición de las oraciones que deben ser leídas y al contenido de las mismas son tenidos en cuenta para este efecto (Daneman y Carpenter, 1980: 452). Operaciones reconstructivas en las que se trata de recordar la última palabra teniendo en cuenta otras palabras de la oración que la contenía, así como algunos errores de selección sobre palabras de la misma oración pero no finales, prueban a posteriori que ha existido procesamiento sintáctico efectivo (Daneman y Carpenter, 1980: 457).

En el segundo experimento de su estudio, Daneman y Carpenter (1980) ofrecen una versión de listening span cuyos resultados correlacionan de forma positiva y significativa con los del reading span. De esta manera los autores concluyen que, en este caso, las diferencias entre medio escrito y medio oral no son relevantes. En la prueba de listening span a los sujetos se les pide recordar la última palabra de oraciones que deben escuchar y no leer. Para garantizar en este caso que el recuerdo de la última palabra se asocia a un procesamiento sintáctico efectivo se introduce la novedad de solicitar al sujeto un juicio de veracidad sobre las oraciones que escucha, las cuales serían falsas en un $50 \%$ de los casos. Las respuestas de los sujetos a estos juicios de veracidad de las oraciones no son tenidas en cuenta a la hora de puntuar los resultados. Se entiende, en cualquier caso, que el mismo hecho de tener que establecer el juicio implica de por sí una actitud activa de procesamiento sintáctico y hace que la retención de la última palabra de las oraciones no se transforme en una operación automatizada de memorización de palabras desligadas de contexto sintáctico.

A partir de la propuesta de Daneman y Carpenter (1980) han sido numerosos los estudios que han utilizado la versión original o versiones adaptadas de reading span o listening span para la evaluación de capacidades de memoria de trabajo en diferentes ámbitos aplicados. La evaluación de la comprensión lectora para sujetos sin lesión cognitiva ha sido uno de ellos, pero versiones adaptadas de listening span para la evaluación de capacidades de memoria de trabajo en sujetos con afasia han sido también propuestas por Caspari et al. (1998) y Ivanova y Hallowell (2014). Pruebas de reading span o listening span se contienen, a veces, como tareas específicas dentro de baterías de evaluación de habilidades cognitivas generales, como es el caso de López-Higes et al. (2012).

El uso de este instrumento como predictor de calidad en la práctica interpretativa tiene también sus antecedentes. Podemos citar como referentes pioneros para el uso de una prueba de reading span a la hora de evaluar capacidades cognitivas de los intérpretes los estudios de Darò y Fabbro (1994), y de Padilla Benítez (1995). Versiones adaptadas de listening span para la evaluación de capacidades de memoria de trabajo en intérpretes son propuestas por Liu et al. (2004) y por Nordet y Voegtlin (1998). Esta última propuesta es también, por otra parte, la que se maneja en el estudio anteriormente referido de Köpke y Nespoulous (2006). En todos estos estudios se entiende que la adaptación de las pruebas de reading span o de listening span sirve para adecuarlas al tipo de sujetos a los que se administra y garantiza, con diferentes tipos de criterio, que la operación de memorización de la última palabra de las ora- 
418 ciones leídas o escuchadas implica algún tipo de procesamiento sintáctico efectivo.

\section{PROPUESTA DE VERSIÓN ADAPTADA DE UNA PRUEBA DE LISTENING SPAN, COMO INDICADOR DE CALIDAD EN LA PRÁCTICA INTERPRETATIVA}

Nuestro objetivo ahora es presentar las características de una prueba de listening span adaptada, que sirva como indicador, entre otros, de la calidad en la práctica interpretativa de estudiantes que se inician en esta actividad. Ha sido usada inicialmente en el estudio experimental de Anssari-Naim (2021), en el que se sometía a evaluación, como se ha dicho anteriormente, el peso relativo de factores cognitivos y rasgos de personalidad en aprendices de interpretación. La memoria de trabajo se ha considerado un componente central de los factores cognitivos y la prueba de listening span se ha seleccionado como instrumento representativo de las capacidades de memoria de trabajo. Los resultados de este estudio ofrecen correlaciones positivas y estadísticamente significativas entre listening span y una prueba con la que se mide calidad de la práctica interpretativa, con los matices que se comentan más adelante.

La prueba de listening span tiene en cuenta el proceder de Daneman and Carpenter (1980), pero el referente más inmediato que se utiliza es la versión ofrecida por López-Higes et al. (2012), donde una prueba de reading span se incorpora, como se ha mencionado, dentro de una batería más general de evaluación de capacidades cognitivas en sujetos con diferentes grados de deterioro cognitivo. La prueba de listening span adaptada a partir de López-Higes et al. (2012) incrementa grado de dificultad y especificidad en tanto que instrumento medidor de eficiencia en la práctica interpretativa. El motivo por el que se selecciona la versión de López-Higes et al. (2012) como referente tiene que ver con el tipo de proyecto de investigación en el que se inscribe el presente estudio (véase nota 1). En este proyecto se evalúan capacidades metacognitivas o de funcionamiento ejecutivo, como la memoria de trabajo o la atención, asociadas al uso de lenguaje en tres tipos genéricos de situación: la del deterioro cognitivo en afasias o demencias, la de la adquisición de primera lengua, y finalmente también la situación propia de tareas relacionadas con las prácticas interlingüísticas. Dentro de estas últimas, la práctica interpretativa representaría una condición de máxima exigencia sobre el control metacognitivo del lenguaje, y ofrece así un polo de contraste que se ha considerado muy relevante en el referido proyecto. Disponer de instrumentos de exploración compartidos y con resultados estandarizados es, sin duda, necesario para hacer comparables los resultados. Ello permitiría, por ejemplo, determinar si existen o no diferencias significativas en memoria de trabajo entre sujetos ejercitados en tareas de interpretación simultánea, y sujetos control de idéntico rango de edad, aunque esta cuestión en concreto queda fuera de los objetivos del presente estudio.

En Anssari-Naim y Hernández (2019) se da cuenta de los aspectos o dimensiones que se han tenido en cuenta para la adaptación de la prueba de reading span contenida en López-Higes $e t$ al. (2012). Antes de nada, la prueba correspondiente de listening span se ofrece con frases de elaboración propia, a cuyas características nos referimos de inmediato. Se incrementa, por otra parte, el grado de exigencia en la capacidad retentiva de las últimas palabras de las oraciones que se someten a escucha. En López-Higes et al. (2012) las oraciones que integran la prueba se organizan en bloques que van de 2 a 6 oraciones, en una primera fase de dificultad creciente, y de 6 a 2 , en una segunda fase de dificultad decreciente. 
Se entiende que al sujeto se le asigna como tarea retener y poner por escrito la última palabra de las oraciones que integran cada bloque, cuando finaliza la presentación de las oraciones que integran cada uno de ellos. Tras observar en un estudio piloto realizado con 10 estudiantes de interpretación el poco valor discriminante de la prueba, se decidió incrementar el grado de dificultad sumando dos oraciones a cada uno de los bloques, de manera que la primera fase, de dificultad creciente, presenta bloques que van de 4 a 8 oraciones y la segunda fase, de dificultad decreciente, bloques que van de 8 a 4 oraciones. La prueba de reading span de López-Higes et al. (2012) presenta valor discriminante, pero teniendo en cuenta la variabilidad en la edad de los sujetos examinados y diferentes grados de deterioro cognitivo.

Se observó que para los sujetos examinados esta adición de dos unidades por bloque incrementaba sustancialmente el valor discriminante inter-sujetos de la prueba (véanse más adelante los resultados que lo confirman: distribución estadística normal). Este incremento en el valor discriminante se hacía aún más patente con el sistema de puntuación que se propuso. Este sistema de puntuación consiste en establecer sencillamente el sumatorio de aciertos, esto es, palabras que el sujeto es capaz de retener en el conjunto de la prueba.

Al margen de estos aspectos, lo más significativo de la adaptación propuesta tiene que ver con la especificidad relativa a la práctica de la interpretación. Los sujetos examinados se han iniciado en la actividad interpretativa entre su lengua nativa, español, y su lengua extranjera, francés. Pareció muy relevante, en este sentido, si consideramos la relevancia de la familiaridad con la lengua extranjera para la buena práctica interpretativa, presentar a los sujetos dos versiones de la prueba de retención auditiva de palabras, una en lengua nativa y otra en lengua extranjera. Se tuvieron en cuenta para ello algunos resultados como los contenidos en Jiménez Ivars et al. (2014). En este estudio se observa que las correlaciones positivas significativas entre una prueba de auto-eficacia y una prueba con la que se mide eficiencia y calidad en la interpretación solo se manifiestan en sujetos con un conocimiento medio-alto de lengua extranjera. Lo afirmado para una prueba que mide un rasgo de personalidad, como la auto-eficacia, entendemos que puede trasladarse a cualquier otra prueba en la que se examinan variables cognitivas. Para el caso concreto de la memoria de trabajo y sus relaciones con el nivel de conocimiento de lenguas en la práctica interpretativa contamos también con el antecedente de Tzou et al. (2012). Sin determinado nivel de conocimiento de la lengua extranjera carece de fundamento examinar la supuesta relación entre capacidades de memoria de trabajo y actividad interpretativa. Las dos versiones lingüísticas de la prueba se ofrecen, tal como se propusieron a los sujetos examinados, en el Anexo I de este estudio.

\section{GARANTÍAS SOBRE EL PROCESAMIENTO LINGÜÍSTICO EN EL DISEÑO DE LA VER- SIÓN ADAPTADA}

Como se ha dicho, una precaución especial manifestada ya en Daneman y Carpenter (1980) es garantizar que la operación sometida a exploración sea efectivamente una tarea de memoria de trabajo y no una simple operación de memoria a corto plazo. Para ello, debe garantizarse que la operación de retención de la última palabra de las oraciones sometidas a lectura o escucha se encuentre asociada a algún tipo de procesamiento lingüístico. Desde nuestro punto de vista, este procesamiento puede incluir cualquier nivel de organización del sistema 
420 lingüístico, no solo el propiamente sintáctico, sino también el semántico, el morfológico, el léxico e incluso el fonológico.

En la versión adaptada que se propone esto último se ha querido garantizar evitando lo que consideramos "artefactos", como ofrecer la mitad de oraciones falsas $\mathrm{u}$ oraciones sin sentido, para solicitar a los sujetos juicios de veracidad o de sentido que pueden ser problemáticos, aparte de difíciles de integrar en el sistema de puntuación. Se ha preferido ofrecer oraciones que describen escenas comunes cuyo ámbito referencial puede resultar familiar o es fácil de imaginar por los sujetos examinados. Ello propicia una actitud natural en el procesamiento del material lingüístico que se ofrece. Esto es, nos referimos a un tipo de actitud que no encapsula la formulación de la oración desligándola del entorno funcional que se supone que motiva su uso. Esto nos parece fundamental para garantizar que existe un procesamiento lingüístico efectivo.

Adicionalmente, y para evitar el efecto de previsión sobre el momento en que aparecerá la última palabra de cada oración, en el diseño de la prueba se ofrecen oraciones con un número de palabras variable. Los rangos de variación de longitud de las oraciones en número de palabras van de 5 a 17 palabras en la versión española y de 7 a 18 en la versión francesa. Esta circunstancia propicia la actitud de procesamiento semántico, esto es, de verdadera actividad de comprensión de lo que se oye. Aunque Daneman y Carpenter (1980) incluyen también oraciones de longitud variable, otras pruebas adaptadas de reading span o listening span tienden a uniformar la longitud de las oraciones, lo que parece un error de diseño de una prueba destinada a evaluar memoria de trabajo. Por otra parte, las garantías de un procesamiento semántico asociado a la tarea se consiguen proponiendo junto a oraciones simples otras compuestas por yuxtaposición, coordinación o subordinación. Sobre todo en el caso de la yuxtaposición, la garantía de la conexión, aparte del recurso entonativo, exige claramente la conexión semántica. Las oraciones que se ofrecen se encuentran desligadas semánticamente, pero no así las cláusulas que integran cada oración.

\section{5. ÍNDICES DE PROCESAMIENTO LINGÜÍS- TICO QUE SE OFRECEN TRAS EL ANÁLISIS DE ERRORES EN LOS RESULTADOS}

\subsection{Efecto bañera}

Ha resultado muy interesante observar cómo en gran medida el contraste entre palabras recordadas y palabras no recordadas manifiesta un efecto bañera (bathtub effect) (Aitchison, 1989) en cada una de las series, de manera que las palabras finales de las primeras o últimas oraciones de cada serie tienden a ser mejor recordadas que las palabras finales de oraciones que aparecen en posiciones intermedias. En series de 7 y 8 oraciones este efecto puede tener una doble manifestación con algún pico en la parte central de las series. Esto no deja de ser un efecto genérico ya señalado por Aitchison (1989), aunque referido más específicamente a sílabas iniciales o finales de una palabra que trata de recordarse. Dado el orden lineal del discurso, la pregnancia perceptiva de lo que aparece al principio o al final de una secuencia lingüística parece clara. Figuras retóricas como la anáfora o la epífora lo acreditan, así como, en particular, para la posición final sucede con la rima. Ignoramos la relevancia última de este efecto, y el grado de especificidad lingüística del mismo, pero en todo caso es un aspecto no extrapolable al procesamiento de estímulos visuales, que tiende a focalizar el centro de un espacio perceptivo con la calidad de figura y no 


\begin{tabular}{|c|c|c|c|c|c|c|c|}
\hline Serie 1 & 15 & 14 & 13 & 17 & & & \\
\hline Serie 2 & 12 & 13 & 12 & 5 & 8 & & \\
\hline Serie 3 & 12 & 13 & 10 & 15 & 5 & 14 & \\
\hline Serie 4 & 14 & 10 & 14 & 4 & 8 & 9 & 13 \\
\hline Serie 5 & 14 & 12 & 8 & 8 & 5 & 6 & 11 \\
\hline Serie 6 & 9 & 8 & 10 & 8 & 5 & 1 & 3 \\
\hline Serie 7 & 14 & 9 & 9 & 11 & 6 & 9 & 16 \\
\hline Serie 8 & 9 & 10 & 15 & 1 & 8 & 17 & \\
\hline Serie 9 & 10 & 12 & 9 & 11 & 12 & & \\
\hline Serie 10 & 15 & 11 & 9 & 15 & & & \\
\hline
\end{tabular}

FIGURA 1. Efecto bañera en versión francesa de la prueba

\begin{tabular}{|c|c|c|c|c|c|c|c|c|}
\hline Serie 1 & 15 & 14 & 15 & 17 & & & & \\
\hline Serie 2 & 13 & 16 & 13 & 15 & 14 & & & \\
\hline Serie 3 & 14 & 16 & 10 & 15 & 12 & 15 & & \\
\hline Serie 4 & 14 & 11 & 14 & 7 & 8 & 12 & 16 & \\
\hline Serie 5 & 15 & 15 & 13 & 9 & 7 & 10 & 6 & 16 \\
\hline Serie 6 & 10 & 11 & 13 & 11 & 10 & 10 & 6 & 13 \\
\hline Serie 7 & 16 & 13 & 13 & 11 & 11 & 14 & 14 & \\
\hline Serie 8 & 13 & 12 & 9 & 13 & 13 & 17 & & \\
\hline Serie 9 & 13 & 15 & 15 & 15 & 14 & & & \\
\hline Serie 10 & 12 & 16 & 15 & 16 & & & & \\
\hline
\end{tabular}

FIGURA 2. Efecto bañera en versión española de la prueba

los márgenes, que suelen procesarse como fondo. En cualquier caso, podemos entender que el efecto bañera representa un patrón de memorización congruente con el orden lineal del discurso, aplicable en la prueba de listening span a la secuencia de oraciones procesadas.

A partir de los resultados del presente estudio, mostramos en las figuras 1 y 2 la forma en que este patrón de memorización se manifiesta, aunque sea solo a modo de tendencia. Los números representan cuántos sujetos (de un total de veinte) han recordado la palabra correspondiente a la posición en que se sitúa el número. Por ejemplo, en la serie 1 de la versión francesa, la primera palabra es recordada por 15 sujetos, la segunda palabra por 14 , la tercera por 13 y la cuarta por 17. De esta manera, la primera y la úl- tima palabra de la serie son las más recordadas. El patrón no se aplica, por supuesto, de forma sistemática, pero una breve inspección de las matrices que aquí presentamos muestra que las palabras recordadas por un menor número de sujetos suelen ocupar posiciones centrales en las series. Por ejemplo, palabras recordadas por $5 \mathrm{o}$ un menor número de sujetos nunca se encuentran en posiciones marginales de inicio o final de serie en la matriz de resultados correspondiente a la versión francesa. Lo mismo puede decirse para palabras recordadas por 10 o un número menor de sujetos en la matriz correspondiente a la versión española. Téngase en cuenta que el número de aciertos para la versión española de la prueba es globalmente superior al número de aciertos para la versión francesa. 


\section{5.2. Tipos de error como indicativos de proce- samiento lingüístico}

El análisis de los errores concretos realizados por los sujetos a la hora de tratar de recordar una palabra serviría como indicio de que el procesamiento lingüístico se encuentra implicado en la operación de memorización. Daneman y Carpenter (1980: 457) ya anticipaban, según se ha dicho anteriormente, este tipo de cuestión cuando afirman lo siguiente:

Finally, it was apparent during some of the recall trials in the span test that subjects sometimes used reconstructive processes. When the subject was unable to produce the sentence final word, he/she might attempt to reconstruct the sentence on the basis of whatever "gist" had been retained. In these reconstructions, subjects sometimes made systematic errors by choosing a word that had been present in the sentence but was not the final word. These informal observations and the subjects' retrospections suggest that the reading span task was successful in taxing the processing and storage capacity of working memory.

Esta nota marginal de Daneman y Carpenter (1980) merece una reflexión adicional. La tipología de errores observados es a nuestro entender indicativa de que en la operación de memorización se encuentran implicados todos los niveles de organización del sistema lingüístico. Estos errores son indicativos de que la tarea realizada por los sujetos no es una simple operación de almacenaje, sino que tiene también que ver con el modo en que globalmente procesamos el lenguaje. Ofrecemos aquí una tipología básica de errores elaborada a partir de las respuestas de los sujetos que participaron en el presente estudio realizando la prueba adaptada de retención auditiva de palabras. Se han considerado tipificables situaciones representadas por cin- co o más ejemplos en cada una de las versiones lingüísticas, salvo para el caso de interferencias asociadas a la homofonía que se observan solo para la versión francesa.

Lematizaciones: Un error común en la operación de memorización consiste en ofrecer una variante en la forma de la palabra, pero dentro del mismo lema o entrada de diccionario. Este tipo de error constituye una desviación fácilmente explicable en términos de procesamiento léxico o, más específicamente, lexicográfico asociado a la operación de memorización. Es el caso de ejemplos como «agria» por «agrias» o «pensar» por «pensando» en la versión española; y de ejemplos como «voter» por «votera» o de «faire» por «fait» en la versión francesa.

\section{Errores asociados al sintagma final de la oración:} Suele ser también común que el sintagma final de una oración interfiera en la operación de memorización de la última palabra, como sucede para los casos que se citan a continuación. Hay aquí un determinante sintagmático del error. Podemos encontrar dos tipos de situación. En algunos casos, se ofrece no la última palabra exactamente, sino esta palabra y alguna otra a la que aparece ligada en el sintagma final, como el artículo o preposición más artículo si se trata de un nombre. Este sería el caso de una respuesta como «un jeu» cuando se pide recordar la última palabra de la oración «Avec un peu $d$ 'inteligence on peut transformer une tâche dificile en un jeu», o el caso de una respuesta como «a las diez» cuando se pide recordar la última palabra de la oración «Se anuncia que la ceremonia tendrá lugar a las diez». La interferencia del sintagma final explica en otros casos que lo que se ofrezca sea una palabra integrada en el sintagma final, pero no la palabra final. Este sería el caso por ejemplo cuando se ofrece como respuesta «cooperar» para la oración «Cuando 
trabajas en una empresa, debes saber cooperar con el grupo», o la respuesta «rencontre» para la oración «A chaque fois que je le voie, je me rappelle de notre première rencontre en 1997» (nota: los números son considerados como palabra, aunque sean un complejo sintagmático).

Palabra final de cláusula, pero no de oración: Cuando las oraciones son compuestas, en ocasiones se ofrece una palabra final de cláusula, pero no de oración. Ello presupone en todo caso interferencia del procesamiento sintáctico en la operación de memorización. Este sería el caso de la respuesta «chien» para la oración «Martine sort promener son chien, peu importe le temps qu>il fait», o la respuesta «sándwich» para la oración «Si no quieres llevarte tu sándwich, puedes comprarlo en el bar».

Perseveraciones sintagmáticas: El error consiste en este caso en ofrecer una palabra perteneciente a la oración, pero que no es palabra final de oración, ni tampoco propiamente final de cláusula. Este sería el caso de la respuesta «sangre» para la oración «La prueba irrefutable que utilizó el juez es la mancha de sangre de la camisa del acusado» o la respuesta «plombier» para la oración «J 'attends le plombier a $18 \mathrm{~h}$, il viendra pour régler le robinet du lavabo». Las perseveraciones ocasionalmente afectan también a palabras que han aparecido en oraciones previamente escuchadas dentro de la misma serie o incluso pertenecientes a series anteriores.

Interferencias con el procesamiento léxico: Las relaciones léxicas que mantiene una palabra con otras o los campos asociativos en los que se inserta constituyen factores particulares de interferencia en la memorización. La palabra que se ofrece como respuesta puede presentar una relación de sinonimia con la palabra objeto de memorización, como sería el caso de «lavabo» por «fregadero», de «mujer» por «esposa», o de «eau» por «débit» (caudal de un río). En ocasiones las relaciones se ofrecen entre merónimos (partes de un todo) como en «frente» por «ojos» o «dientes» por «ojos» (partes de la cara). La relación puede darse también entre antónimos como en «escuchar» por «hablar». Otras relaciones pueden implicar pertenencia a campos asociativos como en «parler» por «penser»o en «ecológicos» por «biológicos». Las interferencias de procesamiento léxico se observan con mucha más frecuencia en las respuestas a la versión española de la prueba que en las respuestas a la versión francesa. Es posible que la lengua nativa sea más propicia a este tipo de interferencias, pero se necesitarían más datos para llegar a una conclusión fundamentada sobre este hecho. Se supone, en cualquier caso, que las conexiones entre elementos léxicos son más intensas y activas para lengua nativa que para lengua extranjera.

Interferencias asociadas a la homofonía: Una reflexión particular merece el caso de la homofonía, que resulta muy frecuente en francés para variantes flexivas de una palabra. Por ejemplo, responder con «plat» en lugar de «plats» o con «film» en lugar de «films» son desviaciones que se encuadrarían dentro del caso general anteriormente consignado como lematizaciones, pero el fenómeno merece un comentario adicional. En la versión francesa de la prueba se contiene, por ejemplo, la oración: «Normalment on ne fait pas attention à ce que 1 ' on peut apprendre à travers les films». Pues bien la identificación de la forma plural «films» exige procesar un elemento del entorno sintáctico como es el artículo en su forma plural «les», que no sería homófono de su forma singular «le». Otros aspectos del entorno sintáctico pueden requerirse para desambiguar palabras homófonas en francés, sean variantes 
424 flexivas como «imagine» $\mathrm{y}$ «imaginent», o unidades léxicas diferentes como «mettre» y «maître». Se consignan errores del tipo «imagine» por «imaginent» $\mathrm{y}$ «mettre» por «maître» respectivamente en las oraciones: «Que fera cet enfant plus tard? Peut-être rien de ce que ses parents imaginent» $\mathrm{y}$ «Le chat sauvé par les pompiers a courru imediatement vers son maître». En estos casos los aciertos, que son mayoritarios, prueban claramente que el procesamiento sintáctico se ha implicado en la operación de memorización.

Errores combinados: En ocasiones los errores observados pueden implicar a más de un tipo de los que acabamos de consignar. Este sería el caso de una respuesta como «tribunal» cuando se pide recordar la última palabra de la oración: «La prueba irrefutable que utilizó el juez es la mancha de sangre de la camisa del acusado». En la respuesta: «tribunal», que ofrece uno de los sujetos, se combinan dos errores. Por un lado, lo recordado parece referirse a una palabra final de cláusula, pero no de oración. Por otra lado, parece existir una interferencia basada en una relación léxica asociativa («tribunal» por «juez»). Los casos de errores combinados aparecen normalmente en respuestas singulares de algún sujeto, pero son difícilmente sistematizables.

En definitiva, de forma directa o indirecta, el análisis de errores revela que la operación de memorización de la última palabra de las oraciones integrantes de la prueba de retención auditiva se encuentra asociada a otras operaciones de procesamiento lingüístico. Esto puede afirmarse al menos en una visión global de los hechos, aunque algunas respuestas individuales, y solo para algunas oraciones, puedan contradecir esta regla general.

\section{ESTUDIO EXPERIMENTAL}

La prueba adaptada de retención auditiva de palabras, cuyas características acabamos de someter a análisis, se administró a 20 sujetos, estudiantes que se inician en la práctica de la interpretación del grado en Traducción y Mediación Interlingüística de la Universidad de Valencia. Los resultados de la versión española (RETEN.Es) y la versión francesa (RETEN.Fr) de la prueba se correlacionaron con los resultados de una prueba ${ }^{4}$ con la que se evalúa una práctica de interpretación simultánea francés-español de un vídeo (https:// www.youtube.com/watch?v=5twQV3G6yIo) de 4 minutos de duración que versaba sobre temática relacionada con los estudios de interpretación (INTERPR.) El sistema de puntuación de esta prueba es el estandarizado en la guía docente de la asignatura de interpretación. Todos los sujetos cuentan con un nivel $\mathrm{C} 1$ acreditado de conocimiento en lengua extranjera francés.

Los resultados de las tres pruebas mostraron, sobre 20 sujetos, una distribución estadística normal con prueba Kolgomorov-Smirnov. Presentaron los siguientes descriptivos estadísticos:

Tabla 1

\begin{tabular}{llll} 
& N & Media & DT \\
\hline INTERPR. & 20 & 4.7 & 1.102 \\
\hline RETEN.Fr & 20 & 33 & 9.666 \\
\hline RETEN.Es & 20 & 43 & 10.050 \\
\hline
\end{tabular}

4 Se evaluó la calidad de la interpretación en una escala de 0 a 10, que se transforma en cualificación académica del estudiante, siguiendo los criterios fijados en la guía académica de la asignatura que cursan. Se toman en cuenta los siguientes factores de evaluación: claridad en la vocalización, adaptación de la entonación a los contenidos, pausas, vacilaciones, corrección gramatical, adecuación léxica, coherencia y estilo del discurso y fidelidad al contenido del discurso original. 
Las correlaciones entre resultados se consignan en la tabla 2.

Tabla 2

\begin{tabular}{llll}
\hline$r_{(x, y)}$ & INTERPR. & RETEN.Fr & RETEN.Es \\
\hline INTERPR. & 1 & & \\
\hline RETEN.Fr & $.617^{* *}$ & 1 & \\
\hline RETEN.Es & .396 & $.798^{* *}$ & 1 \\
\hline
\end{tabular}

** Correlación significativa a nivel de confianza .01

Aunque todas las correlaciones resultaron positivas conviene diferenciar las siguientes circunstancias. Se observó que respecto a la prueba de interpretación solo la capacidad retentiva en francés alcanzó un valor correlacional estadísticamente significativo $\left(.617^{* *}\right)$. La correlación respecto a la capacidad retentiva en español es positiva, pero estadísticamente no significativa (.396). Esto llevó a considerar la especial relevancia de evaluar capacidades de memoria de trabajo en lengua extranjera cuando el objetivo es predecir calidad o eficiencia en la práctica interpretativa. Evaluar capacidades de memoria de trabajo en lengua nativa no deja de ser por ello relevante, al menos para establecer una base de comparación respecto a la misma capacidad en lengua extranjera.

Debe destacarse igualmente que los resultados de capacidad retentiva en español y francés correlacionan de manera positiva y significativa $\left(.798^{* *}\right)$, pero que al mismo tiempo, tras realizar la prueba T-Student sobre los resultados, se observó una diferencia de medias que resultaba también estadísticamente significativa $(t(19)=6,839 ; p=.000)$. En definitiva, los sujetos que mostraron mejores resultados de capacidad retentiva en francés son los que mostraron mejores resultados de capacidad retentiva en español, pero existían diferencias significativas en el grado en el que se manifestaban las capacidades de retención en lengua extranjera y lengua nativa. Esta circunstancia habla, por una parte, de una base genérica común para la capacidad de memoria de trabajo, pero, por otra parte, también de la relevancia que presenta el tipo específico de tarea con el que se somete a examen esta capacidad.

\section{CONCLUSIONES}

Tanto los resultados del estudio experimental como la fundamentación metodológica que precede pensamos que justifican la validez de la prueba de retención auditiva de palabras que aquí presentamos en tanto que indicador de calidad en la práctica interpretativa. Al menos, puede afirmarse esto para el tipo de sujetos sometidos a examen, estudiantes que se inician en la práctica interpretativa. Aunque la tarea que se solicita de los sujetos supone un tipo de actividad desligada de lo que supone la práctica interpretativa real, los resultados que ofrece la prueba de retención auditiva de palabras resultan ser indicios significativos del tipo de habilidades que tienen que poner en juego los estudiantes que se enfrentan a la práctica de la interpretación en las primeras etapas formativas.

Podría sugerirse incluso que esta $u$ otras tareas de retención auditiva de palabras se integraran como actividades en las clases de interpretación, ya que servirían para reforzar capacidades de memoria ligadas a procesos de atención selectiva, algo fundamental para la buena práctica de la interpretación. El análisis de los errores cometidos en la tarea de retención de palabras serviría también para predecir el tipo de interferencias más comunes con las que se enfrenta un intérprete, esto es, el tipo de estímulos que debe someter a control o el tipo de respuestas que debe inhibir. 
Naturalmente, para asegurarnos de su validez, la prueba deberá replicarse con un mayor número de sujetos y será interesante también realizar estudios longitudinales que tengan en cuenta diferentes fases formativas en la práctica de la interpretación, o comparar resultados sometiendo a examen a intérpretes profesionales. Por otra parte, dada la relevancia que parece presentar el factor lengua extranjera como determinante específico de las capacidades de memoria de trabajo, deberá incluirse en futuros estudios una valoración más ajustada sobre el conocimiento de la misma. Parece en cualquier caso muy relevante que en las pruebas de retención auditiva de palabras, y previsiblemente en otras pruebas con las que se evalúan capacidades de memoria de trabajo, se propongan versiones en lengua nativa y extranjera, si lo que se quiere es que los datos obtenidos tengan algún tipo de capacidad predictiva sobre la calidad de la práctica interpretativa.

Debe señalarse, finalmente, que explorar la dimensión metacognitiva de la práctica de la interpretación (Aguirre Fernández-Bravo, 2015) supone tener en cuenta un conjunto complejo de variables que interactúan entre sí. La viabilidad de cualquier estudio que se haga sobre el tema exige diferenciar alguna o algunas de ellas, como se ha hecho en este estudio respecto a una tarea específica de retención de palabras. Este proceder obligado para cualquier labor de investigación no debe hacer olvidar que lo que se concluya debe ser siempre valorado dentro de un contexto mucho más amplio de consideraciones. En el caso concreto de la práctica de la interpretación, el peso relativo de factores cognitivos y rasgos de personalidad podría ser diferente a lo largo de las etapas formativas, o cuando se somete a comparación la práctica de los estudiantes con la de los profesionales de la interpretación.

\section{REFERENCIAS}

Aguirre Fernández-Bravo, Elena. (2015): El desarrollo metacognitivo del estudiante de interpretación: Estudio de caso, Madrid: Universidad Pontificia de Comillas.

Aitchison, Jean (1989): Words in the Mind: An introduction to the mental lexicon, Cambridge, Massachusetts: Blackwell.

AnsSARI-NAIM, Saida (2021): «A comparative assessment of listening span and self-efficacy in simultaneous interpreting training», Sendebar, 32, 167-176.

AnsSARI-NAIM, Saida y Carlos Hernández Sacristán (2019): «Adaptación de un test de retención auditiva de palabras ('listening span') como indicador de eficiencia en la práctica interpretativa», comunicación presentada al VII Congreso Internacional Entreculturas, Málaga.

BAdDeley, Alan D. (1986): Working Memory, Oxford: Oxford University Press.

BADDELEY, Alan D. (1996). «Exploring the Central Executive», Quarterly Journal of Experimental Psychology, 49A, 5-28.

BADDEley, Alan D. (2012): «Working Memory: Theories, models, and controversies» Annual Review of Psychology, 63(1), 1-29.

BADDELEy, Alan, Eysenck, Michael W. y Anderson, Michael C. (2009): Memory, Nueva York: Psychology Press.

BAdDELEY, Alan D. y Graham Hitch (1974): «Working Memory», en Gordon H. Bower (ed.), The Psychology of Learning and Motivation, Nueva York: Academic Press, 47-89.

BANdura, Albert (1995): Self-Efficacy in Changing Societies, Cambridge: Cambridge University Press.

CASPARI, Isabelle, Stanley R. Parkinson, Leonard L. LaPointe, y Richard C. Katz (1998), «Working Memory and Aphasia», Brain and Cognition, 37, $205-$ 223.

Christoffels, Ingrid (2004): Cognitive Studies in Simultaneous Interpreting, Ipskamp/Enschede: PrintPartners.

Daneman, Meredith y Patricia A. Carpenter (1980), «Individual Differences in Working Memory and 
Reading», Journal of Verbal Learning and Verbal Behavior, 19/4, 450-466.

DARò, Valeria y Franco Fabbro (1994): «Verbal Memory during Simultaneous Interpretation: Effects of phonological interference», Applied Linguistics, 15/4, 365-381.

Hittunen, Sinikka, Rauni Pääkkönen, Gun-Viol Vik, y Christina M. Krause (2016): «On Interpreters' Working Memory and Executive Control», International Journal of Bilingualism, 20/3, 297-314.

HoDÁKovÁ, Soňa (2009): Pamät'v simultánnom a konzekutívnom tlmočení [Memory in simultaneous and consecutive interpreting], Tesis doctoral inedita, Nitra, Konštantín Filozof University.

IvANOVA, Maria V. y Brooke Hallowell (2014): «A new Modified Listening Span Task to Enhance Validity of Working Memory Assessment for People with and without Aphasia», Journal of Communication Disorders, 52, 78-98.

JimÉnEZ IVARs, Amparo, Daniel Pinazo Calatayud y Marta Ruiz i Forés (2014): «Self-efficacy and Language Proficiency in Interpreter Trainees», The Interpreter and Translator Trainer, 8/2, 167-182.

KÖPKE, Barbara y Jean-Luc Nespoulous (2006): «Working Memory Performance in Expert and novice interpreters», Interpreting, 8/1,1-23.

LEE, Sang-Bin (2014): «An Interpreting Self-Efficacy (ISE) Scale for Undergraduate Students Majoring in Consecutive Interpreting: Construction and preliminary validation», The Interpreter \& Translator Trainer, 8, 183-203.

Liv, Minhua, Diane L. Schallert y Patrick J. Carroll (2004): «Working Memory and Expertise in Simultaneous Interpreting», Interpreting, 6/1, 19-42.

LóPEZ-Higes, Ramón, Susana Rubio, María Teresa Martín-Aragoneses, David Del Río y Gema Mejuto (2012): «Assessment of Grammatical Comprehension in Normal and Pathological Aging: A summary of the results obtained with ECCO and ECCO_Senior tests», International Journal of Psychological Research, 5/1, 94-106.

NoRDET, Lilian y Lara Voegtlin (1998): Les tests d'aptitude pour l'interpétation de conference. La mémoire, Mémoire pour l'obtention du Diplôme de Traducteur, Ginebra: Université de Genève.
Padilla Adamuz. Francisca M. (2002): Memoria de trabajo y funciones de control en la interpretación de lenguas, Granada: Universidad de Granada.

Padilla Benítez, Presentación (1995). Procesos de memoria y atención en la interpretación de lenguas, Tesis Doctoral, Universidad de Granada.

Padilla, Presentación; María Teresa Bajo, JoséJuan Cañas, and Francisca Padilla (1995): «Cognitive Processes of Memory in Simultaneous Interpretation», en Jorma Tommola (ed.), Topics in Interpreting Research, Turku: University of Turku Press, 61-71.

TIMARovÁ, Šárka (2008) : «Working Memory and Simultaneous Interpreting», en Pieter Boulogne (ed.), Translation and Its Others. Selected Papers of the CETRA Research Seminar in Translation Studies 2007, http://www.kuleuven.be/cetra/papers/papers.html, 1-28.

TIMARovÁ, Šárka, Ivana Čeňková, Reine Meylaerts, Erik Hertog, Arnaud Szmalec y Wouter Duyc (2014): «Simultaneous Interpreting and Working Memory Executive Control», Interpreting, 16/2, 139-168.

Turner, Marilyn L. y Randall W. Engel (1989): «Is Working Memory Task Dependent?», Journal of Memory and Language, 28/2, 127-154.

Tzou, Yeh-Zu, Zohreh R. Eslami, Hsin-Chin Chen y Jyotsna Vaid (2012): «Effect of Language Proficiency and Degree of Formal Training in Simultaneous interpreting on Working Memory and Interpreting Performance: Evidence from Mandarin-English speakers», International Journal of Bilingualism, 16/2, 213-227.

\section{ANEXO I}

Los bloques de oraciones se designan como series. La prueba se administra en el orden crecientedecreciente de dificultad tal como se ofrece seguidamente. Se señala en cursiva la última palabra de cada oración, sobre la que se solicita memorización. Cada vez que finaliza una serie, el sujeto examinado tiene la indicación de anotar por escrito las palabras recordadas. El tiempo aproximado de administración de la prueba es de 15 minutos. 


\section{PRUEBA DE RETENCIÓN} AUDITIVA EN ESPAÑOL

\section{SERIE 1}

Después de la operación, los médicos le recomendaron que se quedara en cama.

El chico se estaba levantando de la cama cuando vio a su madre .

No quería romper el vaso, se me resbaló de las manos. Los mejores momentos de la vida se pueden resumir en una carta.

\section{SERIE 2}

Normalmente no prestamos atención a lo que nos pueden enseñar las películas.

Es muy agradable despertarse y encontrar el desayuno servido.

No podré cenar contigo; voy a ir al teatro con mi esposa.

A pesar de que ha nevado mucho, los ríos no tienen mucho caudal.

Le sorprendió que su esposa volviera tan tarde.

\section{SERIE 3}

A veces, la gente nos hace decir lo que no hemos dicho.

Podríamos caminar por la playa y disfrutar de la luz de la luna.

Tienes que saber cómo controlarte cuando estás enfadado.

Nadie le gustó la nueva casa de Lola.

La razón por la que he cambiado de trabajo fue para recuperar el aliento.

Algunas personas no necesitan hablar, lo dicen todo con los ojos.

\section{SERIE 4}

La policía persiguió al ladrón durante horas antes de arrestarlo.
Cuando trabajas en una empresa, debes saber cooperar con el grupo.

El París de hoy día no tiene nada que ver con el París de 1920.

Los chicos rompieron el parabrisas del coche rojo aparcado allí justo enfrente.

Me encanta el campo en primavera con todos los campos cubiertos de flores.

La mayoría de los programas de la tarde son para niños.

En la actualidad, los periodistas pasan mucho tiempo hablando, pero no pensando.

\section{SERIE 5}

El gato rescatado por los bomberos corrió inmediatamente hacia su amo.

Espero al fontanero que viene a las 6:00 a poner el grifo del fregadero.

Con un poco de inteligencia podemos transformar una tarea difícil en un juego.

Justo después del primer salto del caballo, el jinete cayó al suelo.

Hay que escuchar la radio para saber lo que está sucediendo ahora en el mundo.

Es un placer almorzar en el jardín mientras se escucha música clásica.

Cuando te vas de vacaciones solo, tarde o temprano sufres de soledad.

Si no quieres llevarte tu sándwich, puedes comprarlo en el bar.

\section{SERIE 6}

Aunque no fuera ya rico, mantenía sus buenos modales.

La prueba irrefutable que utilizó el juez es la mancha de sangre de la camisa del acusado.

En otoño es agradable caminar sobre las hojas secas.

Es normal que haya vínculos muy fuertes entre los miembros de una familia. 
Después del escándalo público, el Primer Ministro probablemente tendrá que dimitir.

Ocho multiplicado por 5 son cuarenta.

El detective siguió al sospechoso hasta la estación, donde se perdió entre la multitud.

Sigue trabajando como conductor para su hermano aunque lo maltrate.

\section{SERIE 7}

Cada vez que lo veo, recuerdo nuestro primer encuentro en 1997.

La vida es un misterio que hay que vivir y no un problema por resolver.

No puedes recuperar el tiempo perdido.

Poder encontrar la alegría en la alegría del otro es el secreto de la felicidad.

Como nadie le hacia caso, se marchó.

Tu marido engordará si le preparas demasiadas comidas buenas.

Porque eres un buen conductor, te crees a salvo de los accidentes.

\section{SERIE 8}

Mientras usted se relaja, su banco trabaja para usted. Me encontré con Roberto; volvía de vacaciones.

No reconocí a tus hijos; han crecido mucho.

Las cerezas que recogiste estaban agrias.

A nadie le gusta esta sopa; tiene sabor a quemado.

Atrapé la rata que se comía mi queso.

\section{SERIE 9}

Aún no sabe por qué candidato va a votar.

¿Qué hará este niño más tarde? Posiblemente nada de lo que sus padres se imaginan.

Se anuncia que la ceremonia tendrá lugar a las diez. Me dice que cuando tenga una mejor situación, comprará un coche .

María vigila todo lo que sus hijos hacen.

\section{SERIE 10}

Juan solo come productos biológicos.

Con mis esquíes nuevos, me caigo todo el tiempo.

Carmen sale a pasear con su perro haga el tiempo que haga.

El director informó a Isabel y Marta de su retraso.

\section{PRUEBA DE RETENCIÓN AUDITIVA EN FRANCÉS}

\section{SERIE 1}

Après son opération, les médecins lui ont recommandé de rester au lit.

Le garçon était en train de descendre du lit quand il a vue sa mère.

Je ne voulais pas casser le verre, il a juste glissé entre mes mains.

Les meilleurs moments de la vie peuvent être recueillis dans une lettre.

\section{SERIE 2}

Normalement on ne fait pas attention à ce que 1 'on peut apprendre à travers les films.

$C^{\prime}$ 'est très agréable de se réveiller et trouver le petit déjeuner servi.

Je ne pourrai pas dîner avec vous or je dois aller au théâtre avec ma femme.

Malgré qu'il a beaucoup neigé, les rivières $n$ 'ont pas beaucoup de débit.

Il était surpris que sa femme revienne si tard.

\section{SERIE 3}

Parfois, les gents nous font dire ce qu ' on n 'a pas dit. On pourrait marcher sur la plage et se réjouir du clair de la lune.

Il faut savoir se contrôler quand on est furieux.

Personne n 'a aimé la nouvelle maison de Brigitte.

La raison pour laquelle $\mathrm{j}$ ' ai changer de travail était pour reprendre du souffle. 
430 Certaines personnes $\mathrm{n}$ ' ont pas besoin de parler, elles disent tout avec leurs yeux.

\section{SERIE 4}

Les policiers ont chassé le voleur pendant des heures avant de pouvoir l'arrêter.

Quand on travaille dans une entreprise, il faut savoir coopérer avec le groupe.

Le Paris d 'aujourd 'hui n 'a rien avoir avec le Paris de 1920.

Les garçons ont cassé le pare-brise de la voiture rouge stationné juste là en face.

$\mathrm{J}$ 'aime bien la campagne au printemps avec tous les champs couverts de fleurs.

La plus part des émissions dans 1 ' après midi sont pour les enfants.

Dans 1' actualité, Les journalistes consacre beaucoup de temps à parler mais pas à penser.

\section{SERIE 5}

Le chat sauvé par les pompiers a couru immédiatement vers son maître.

$\mathrm{J}$ 'attends le plombier a $18 \mathrm{~h}$, il viendra pour régler le robinet du lavabo.

Avec un peu d'intelligence on peut transformer une tâche difficile en un jeu.

Juste après le premier saut du cheval le cavalier est tombé par terre.

Il faut écouter la radio pour savoir ce que se passe immédiatement dans le monde.

Ça fait plaisir déjeuner dans le jardin en écoutant de la musique classique.

Quand on part en vacances tout seul tôt ou tard on souffre de la solitude.

Si vous ne voulez pas prendre votre sandwich, vous pouvez vous en acheter un au bar.

\section{SERIE 6}

Même si il n'est plus riche il a gardé ses bonnes manières.
La preuve irréfutable que le juge a utilisée est la tâche de sang de la chemise de l'accusé.

En automne c' est agréable de marcher sur les feuilles sèches.

$C^{\prime}$ est normal qu'il y ait des liens très forts entre les membres d ' une famille.

Après le scandale public du premier ministre, il doit démissionner probablement.

Huit multiplié par 5 font quarante.

Le détective a suivit le suspect jusqu 'à la gare ou il $\mathrm{s}$ ' est perdu entre la foule.

Il travaille toujours comme chauffeur pour son frère même si il le maltraite.

\section{SERIE 7}

A chaque fois que je le voie, je me rappelle de notre première rencontre en 1997.

La vie est un mystère qu 'il faut vivre et non un problème à résoudre.

On ne peut pas récupérer le temps perdu.

Etre capable de trouver sa joie dans la joie de 1 ' autre est le secret du bonheur.

Comme personne ne lui prêtait pas attention, il est parti.

Votre mari prendra du ventre si vous lui faites trop de bons petits plats.

Parce que vous êtes un bon conducteur, vous vous croyez à l'abri des accidents.

\section{SERIE 8}

Pendant que vous vous détendez, votre banque travaille pour vous.

J'ai rencontré Durand; il rentrait de vacances.

On ne reconnaît pas vos enfants; ils ont beaucoup grandi.

Les cerises que vous avez cueillies étaient acides. Personne n'aime cette soupe; elle a le goût de brûlé. J'ai pris au piège cette souris qui mangeait mon fromage. 


\section{SERIE 9}

Il ne sait pas encore pour quel candidat il votera.

Que fera cet enfant plus tard? Peut-être rien de ce que ses parents imaginent.

On annonce que la cérémonie aura lieu à dix heures.

Il me dit que dés qu'il aura une meilleure situation, il achètera une voiture.

Christiane surveille tout ce que ses enfants font.

\section{SERIE 10}

Gaston ne mange que des produits biologiques.

Avec mes nouveaux skis, je tombe sans arrêt.

Martine sort promener son chien peu importe le temps qu>il fait.

Le directeur a informé Ariane et Dominique de leur retard. 